\title{
Oliveira, Vítor (2016): Urban Morphology. An Introduction to the Study of the Physical Form of Cities
}

\author{
Cham: Springer International Publishing. = The Urban Book series. XXIII. 192 Seiten, \\ $46 \mathrm{~s} / \mathrm{w}$-Illustrationen, 19 farbige Illustrationen.
}

Michael Wurm ${ }^{1}$ (I)

Online publiziert: 7. Februar 2017

(C) Springer-Verlag Berlin Heidelberg 2017

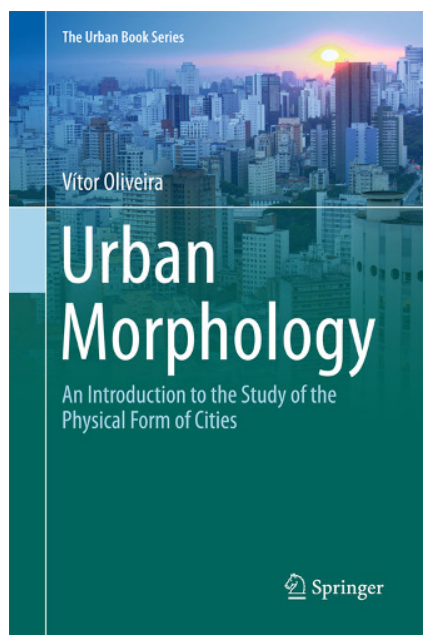

Ein übersichtliches Handbuch über die Wissenschaft der städtischen Form (Urban Morphology) zu verfassen, stellt kein einfaches Unterfangen dar, da es sehr viele Aspekte in sich vereinen soll, welche einerseits einer Auswahl aus dem breiten Spektrum der nicht eindeutig abgrenzbaren Forschungsdisziplin der Urban Morphology bedürfen und zugleich in lehrbuchhafter Manier der interessierten Leserschaft, Studierende und Forschende aus dem breiten Potpourri an wissenschaftlichen Disziplinen wie der Geographie, der Architektur oder der Stadtplanung, die Werkzeuge zur Beschreibung der städtischen Form näherbringen soll. Aber genau dieser Herausforderung hat sich Vítor Oliveira gestellt und mit seinem nach eigener Aussage einzigartigen englischsprachigen Buch „Urban Morphology“ einerseits

Dr. Michael Wurm

michael.wurm@dlr.de

1 Deutsches Zentrum für Luft- und Raumfahrt (DLR), Deutsches Fernerkundungsdatenzentrum (DFD), Münchener Straße 20, 82234 Wessling, Deutschland den vielen Facetten von Städten und ihren Formen einen Rahmen gegeben. In diesem spielt der physische Aspekt der städtischen Form eine Rolle, aber auch die stadtgestaltenden Prozesse und Akteure wollen in Betracht gezogen werden sowie die Einordnung in die Forschungsdisziplin an sich. Für den an städtischen Formen interessierten Leser werden Fäden zu unterschiedlichen Ausprägungen der städtischen Form vorgestellt, welche aufgegriffen werden können, um eine eigene, persönlich gefärbte Reise zur physischen Beschreibung von Städten zu beginnen oder in strukturierter Weise fortzusetzen. Der Anspruch des Buches ist kein geringerer als die Schließung einer vom Autor identifizierten Lücke in der Wissenschaft der städtischen Form (study of urban form: Urban Morphology).

Das Buch ist in zwei Teile mit sieben inhaltlichen Kapiteln gegliedert, die zusätzlich mit einer Einleitung und Schlussfolgerungen abgerundet werden. Eingeleitet wird das Buch durch ein Vorwort von Jeremy Whitehand, dem Vorsitzenden der Urban Morphology Research Group und Herausgeber der Zeitschrift Urban Morphology. Während im ersten Teil (Kapitel 2-5) die Stadt zum zentralen Thema gemacht wird, adressiert der zweite Teil (Kapitel 6-8) Forscher und Anwender.

Der erste Teil befasst sich in Kapitel 2 streckenweise sehr ausführlich mit den Hauptelementen der physischen Stadt, wie sie beschaffen und angeordnet sind. Dabei greift der Autor auf eindrucksvolle und eingängige Beispiele aus verschiedenen bekannten (z.B. Venedig, New York, Barcelona, Paris) und auch weniger geläufigen (z.B. Sanaa, Djenné, Isfahan), jedoch nicht minder interessanten Städten zurück und stellt sie anhand von gut ausgewählten Satellitenbildern dar. Danach widmet er sich den Einflüssen des natürlichen Kontextes auf die städtische Struktur und geht dann sehr ausführlich auf die Hauptelemente der gebauten Stadt ein und wendet sich sehr detailliert und anhand von Beispie- 
len (wiederum mittels Erdbeobachtungsbildern) den Straßen, Plätzen, Baublöcken, Parzellen und Gebäuden zu. Dieses Kapitel stellt eine ungeheure Dichte an Informationen in sehr detaillierter Weise bereit, welche durchaus auch in kompakterer Form dem Leser nähergebracht werden kann, da am Ende vordergründig die Erkenntnis über die enorme Vielfalt der Elemente in der städtischen Formenlandschaft über die Aneinanderreihung der Beispiele in Erinnerung bleibt. In den verbleibenden Kapiteln des ersten Teils werden kurz die Akteure (Stadtplaner, Architekten, Politiker etc.) vorgestellt, die der städtischen Form ihr Gesicht verleihen (Kapitel 3). In Kapitel 4 wird kurz auf die geschichtliche Entwicklung von Städten in unterschiedlichen Perioden (z. B. Mesopotamien, China, Antikes Griechenland, Römisches Reich, islamische Städte, mittelalterliche Städte, Städte der Renaissance und Städte aus dem 19. Jahrhundert) eingegangen. Diese beiden Kapitel werden jeweils kurz und mehr der Vollständigkeit halber abgehandelt, was dem Leser zumindest eine grobe Einordnung gestattet, da die in Kapitel 2-4 eingeführten Werkzeuge in Kapitel 5 anhand von drei Städten wesentlich ausführlicher (New York, Marrakesch und Porto) und mit deutlich anwendungsorientierterem Bezug verwendet werden. Die drei Städte werden anschließend nach den wesentlichen Einflüssen auf die Formgebung der städtischen Struktur dargestellt (individuelle geschichtliche Entwicklung, Agenten und Natur- bzw. Kulturraum). Durch die Unterschiedlichkeit der gut gewählten Beispielstädte wird für den Leser sowohl die Herangehensweise von Urban Morphology deutlich als auch die Bedeutung der Untersuchung der Formen augenscheinlich. Der Leser hat das Gefühl, mehr über diese Städte gelernt zu haben. Die Auswahl der Städte birgt auch eine hohe Wahrscheinlichkeit, in der Zukunft selbst die Brille der Urban Morphology aufzusetzen und diese oder ähnliche Städte mit anderen Augen zu betrachten oder zu bereisen.

Der zweite Teil des Buches widmet sich der für ein Handbuch notwendigen disziplinhistorischen Einordnung der Urban Morphology, greift dabei die anerkannten richtungsweisenden Hauptwerke in der Literatur auf (z. B. Jacobs 1961 und Lynch 1960) und fasst die wesentlichen Aspekte für den Leser zusammen. Kapitel 6 ist hier besonders hervorzuheben - es macht aufgrund seiner Länge den Kern des Handbuches aus -, da hier dem Leser die Möglichkeit gegeben wird, anhand der behandelten Hauptwerke die Entwicklungen der Forschungsdisziplin nachzuvollziehen und eigene Interessen anhand der beschriebenen Forschungsansätze einzuordnen. Gleichzeitig werden die anerkannten morphologischen Ansätze vorgestellt (historicogeographical approach, process typolocial approach, space syntax und spatial analysis) und ausführlich die relevanten Werke und Autoren gewürdigt, wodurch in übersichtlicher Form die Möglichkeit gegeben wird, die Fäden in den Spezialpublikationen aufzugreifen. Gerade diese Übersicht und
Einteilung anhand der bestehenden Literatur kann für Wissenschaftler oder für Studierende eine sehr wertvolle Hilfestellung für einen raschen Einstieg in diese Forschungsdisziplin darstellen, ohne selbst die umfassende Primärliteratur wälzen zu müssen. Als sehr wertvollen Teil von Kapitel 6 kann das letzte Unterkapitel (Kapitel 6.3) gesehen werden, in welchem eine vergleichende Studie zur urbanen Form anhand von einer bereits bekannten Stadt (Porto) detailliert beschrieben wird. Gerade diese Abfolge an theoretischem Lehrbuchwissen und der Anwendung aus wissenschaftlicher Sicht machen das Buch von Oliveira sehr wertvoll. Die letzten beiden Kapitel des zweiten Teils schließen den Kreis und stellen praktische Bezüge der Urban Morphology zu Stadtplanung und Architektur (Kapitel 7) sowie zur sozialen (soziale Gerechtigkeit), wirtschaftlichen (Tourismus) und Umweltdimension (Klimawandel) des städtischen Lebens her (Kapitel 8). Abgerundet wird der zweite Teil des Buches von den Schlussfolgerungen des Autors, in denen er noch einmal auf seine persönliche Motivation zu diesem Buch eingeht und vor allem auf die Relevanz aller unterschiedlichen physischen Elemente verweist sowie die Bedeutung der Akteure für die Gestaltung der Städte und der Urban Morphology herausstreicht.

Das Buch ist in Vollfarbe und reichlich mit Abbildungen illustriert (65 Abbildungen auf 185 Seiten). Gerade die ausgewählten Satellitenbilder stellen einen mittlerweile anerkannten Weg dar, um sich Städten und ihrer physischen Struktur zu nähern. Die Bilder sind in der Regel sehr eindrucksvoll und lehrreich, wenn sie auch an manchen Stellen lediglich zur bildhaften Untermalung der Texte verwendet werden. Aus der Sicht des Lesers wünscht man sich oftmals eine stärkere Verzahnung zwischen Text und Bildern. Dies trifft vor allem auf die Bereiche $\mathrm{zu}$, in denen die Abbildungen aufgrund ihrer geringen Druckqualität (vor allem bei historischen Plänen) und oftmals geringen Größe (bedingt durch das Buchformat) nicht die Wirksamkeit entfalten können, die ihnen eigentlich zugedacht sein mag. Insgesamt ist das Buch ein solides Handbuch zu Urban Morpholo$g y$, es verschafft einen Überblick über alle dazugehörenden Bereiche wie Geschichte, Literatur, Wegweiser oder dem Forschungsobjekt und bietet so Allen (Einsteigern, Studierenden, Forschern) eine ausgezeichnete Orientierung. Das bestehende Wissen wird in diesem Kontext einfach zugänglich gemacht. Dem eigenen Anspruch des Autors, ein Handbuch zu schreiben, das eine von ihm identifizierte Lücke in der Literatur zur städtischen Form schließen soll, wird $U r$ ban Morphology gerecht: Das Buch enthält alle Bestandteile der breit gefächerten Forschungsdisziplin und die Standardwerke dazu werden diskutiert. Ein Buch also, welches Einstiegswerk und Nachschlagewerk zugleich ist, das man gerne als Orientierungshilfe und Wissensquelle zur Hand nimmt. 


\section{Literatur}

Jacobs, J. (1961): The Death and Life of Great American Cities. New York.

Lynch, K. A. (1960): The Image of the City. Cambridge. 